\title{
Generando consensos
}

\author{
Dr. Mario Rodriguez Sammartino \\ Presidente AAOT 2019
}

\section{Estimados consocios,}

Cuando propusimos nuestra candidatura para presidir esta asociación, dijimos que queríamos tratar de unir consensos entre los distintos ortopedistas de todo el país, a través de la elaboración de guías o lineamientos prácticos de las patologías más frecuentes, a fin de permitir que todos los colegas encuentren el respaldo necesario de esta Asociación a la hora de realizar un diagnóstico o tratar a sus pacientes.

Consenso es un término que procede del latín "consensus" y que hace referencia al acuerdo que se alcanza por el consentimiento entre los miembros de un grupo o entre varios grupos.

La complejidad creciente que, en ocasiones, presenta la atención médica y la incertidumbre que esto genera, han propiciado el interés y la incorporación de las llamadas metodologías de consenso, para diagnosticar y tratar a los pacientes en forma racional.

La práctica de la Ortopedia y Traumatología requiere de una permanente toma de decisiones complejas. Frente a un problema musculoesquelético, el ortopedista pone todos sus conocimientos médicos y quirúrgicos, su experiencia y su familiaridad con la literatura, a fin de formular un plan razonable de diagnóstico y tratamiento. Si bien todas estas decisiones se encuentran influenciadas por consideraciones no científicas dependientes del medio, del paciente y hasta de problemas económico-financieros, el ejercicio de la toma de decisiones y su representación gráfica debe ser considerado como una extraordinaria herramienta.

Las guías de práctica clínica (GPC) son recomendaciones desarrolladas de forma sistemática para ayudar tanto al profesional como al paciente a tomar las decisiones adecuadas frente a una enfermedad o condición de salud determinada. La ventaja de estas guías, tanto para el médico como para el paciente, es que están realizadas utilizando la mejor prueba científica existente.

La heterogeneidad en el tratamiento de los pacientes, aun entre especialistas de la misma institución que han recibido entrenamientos similares, es muy grande. Esta situación deriva en una variabilidad muy amplia de criterios diagnósticos, terapéuticos y pronósticos, así como del uso poco eficiente de recursos, y finalmente en un impacto negativo en la calidad de la atención clínica.

Algunas sociedades de ortopedia de países del primer mundo consideran, entre sus prioridades, la elaboración de estas guías para sus asociados y las califican de altamente recomendables para mejorar la calidad médica. Tienen inclusive entidades específicas para la elaboración de estas recomendaciones dentro de un contexto científico altamente calificado. La DGOU (German Society for Orthopaedic and Traumatology) tiene más de 60 GPC elaboradas y puestas a disposición de sus miembros.

Una buena manera de representar las recomendaciones es en forma de algoritmos, que son una representación gráfica en forma de un diagrama de flujo, de un conjunto finito de pasos, reglas o procedimientos lógicos, sucesivos y bien definidos, que se deben seguir para resolver un problema diagnóstico o terapéutico específico. Es una forma de pensamiento secuencial y ordenada para llegar a la solución de un problema.

Tiene que ver también con la "Teoría de la toma de decisiones" de la que tan bien habla Daniel Kahneman, Premio Nobel de Economía, cuando se refiere a las formas de pensamiento y a la heurística como el conjunto de técnicas o métodos para resolver un problema.

Dr. MARIO RODRIGUEZ SAMMARTINO • mariors@infovia.com.ar

Cómo citar este artículo: Rodriguez Sammartino M. Generando consensos. Rev Asoc Argent Ortop Traumatol 2019;84(1):1-2. http://dx.doi.org/10.15417/issn.1852-7434.2019.84.1.947 
Por lo tanto, las GPC:

- Constituyen una poderosa herramienta de integración.

- Ayudan a pensar sistemáticamente.

- Ayudan a la toma de decisiones en situaciones de incertidumbre.

- Ayudan a resolver problemas de variabilidad.

- Mejoran la calidad del ejercicio profesional.

- Unifican los criterios de atención médica institucional.

- Ayudan a la evaluación objetiva de la calidad de la atención médica.

- Favorecen la actualización continua y accesible del conocimiento.

- Promueven el manejo integral del paciente.

- Fomentan la utilización adecuada de las tecnologías médicas.

Durante este año de gestión, trataremos de trabajar en forma conjunta entre nuestro Comité de Educación Médica Continuada y nuestras Sociedades Integrantes para comenzar a elaborar nuestras primeras guías sobre las patologías más frecuentes de cada subespecialidad y poder ponerlas a disposición de nuestros asociados con el fin de generar consensos entre nuestros pares y nuestros pacientes. 